\title{
Human Immuno Deficient Patient Suffering from Exserohilum Rostratum Keratitis
}

\section{Shivam Bajoria and Amrendra Singhania*}

Department College of Paramedical Sciences, Teerthanker Mahaveer University, Moradabad (U.P), India

*Corresponding Author: Amrendra Singhania, Department College of Paramedical Sciences, Teerthanker Mahaveer University, Moradabad (U.P), India.

DOI: 10.31080/ASOP.2020.03.0089
Received: January 20, 2020

Published: January 31, 2020

(C) All rights are reserved by Shivam Bajoria and Amrendra Singhania.

\begin{abstract}
Purpose: To report The prognosis of Exserohilum Rostratum Keratitis In an Immunodeficiency Patient.

Method: Patients History, Clinical Characteristics, Risk factors, Laboratory findings, Treatments and outcomes being kept for review in a retrospective study of keratomycosis caused by E. rostratum in an HIV patient.

Results: An unknown species of insect causes a trauma in the right eye of a 48 year old patient with HIV infection who shows symptoms of blurred vision and redness in the respective eye. Right Cornea shows white infiltrate in the Biomicroscopic examination. Epithelial surface and anterior stroma shows deposition of brownish pigmented deposits, A feathery edge and satellite lesion. $E$. rostratum got identified by Polymerase Chain Reaction (PCR),Large dematiaceous septate hyphae is shown by Corneal scraping Specimen. Primary Treatment include 5\% netamycin eye drops and oral Itraconazole. Corneal Lesion shows good prognosis by the medication provided.

Conclusions: An Immunocompromised Patient with ocular trauma shows Corneal phaeohyphomycosis caused by Exserohilum. Corneal scrapings and Polymerase Chain Reaction diagnosed Exserohilum which causes Brown pigmented lesion in a white infilterate. Keywords: E. rostratum; Exserohilum Rostratum; Cornea
\end{abstract}

The Creative Commons Attribution Non Commercial 4.0 International License (CC BY - NC) licensed the article

Name of investigator

- Winai Chaidaroon.

- Mitt Phaocharoen

- Titipol Srisimboon.

- $\quad$ Nongnuch Vanittanakom

Institution involved in research

- $\quad$ Ching Mai University, Department of Ophthalmology, Faculty of medicine, Ching Mai Thailand.

- Ching Mai University, Ching Mai Thailand, Department of Microbiology, Faculty of medicine.

Rationale and background information

The foremost basis of the project is to enhance the knowledge about the pathogenic nature of exserohilum species on humans. The study tells us about the finding areas of Exserohilum species and about it's nature. The study tells us about the appropriate medication which should be given at the time of ocular infection with the Exserohilum species. The research needs to be done to evaluate the prognosis rate in patient after infection with Exserohilum species.

\section{Exserohilum}

Mainly found in Grass and soil. Three species E. rostratum, E. longirostratum, E. mcginnisii found Infectious to Humans either in a immunocompromised or immunocompetent patient. Rarely found pathogenic to Humans. Human Immunodeficiency Virus: It is a single stranded RNA containing Retrovirus which causes AIDS in humans. This generally shows its action after its activation inside the host.

Study goals and objectives

Some of the important goals for the study are

- What is the prognosis rate of the patient after the infection with Exserohilum species.

- What are the major founding areas of Exserohilum species.

- What is the pathogenic nature of Exserohilum species on humans. 
- What are the appropriate. medication which should be given to the patient after an ocular infection with Exserohilum species.

\section{Study design}

- The Retrospective Case study is a Descriptive study.

- A 48 year old man is taken as a Subject which is the sampling frame of the study.

- While the expected duration of the study is taken to be Two months.

\section{Methodology}

Pthisis Bulbi got reported in a 48 year old male patient due to congenital blindness in his left eye. Foreign body thought to be an insect injured patients right eye while riding a motorcycle 1 month prior to be seen. At the primary hospital eye irrigation and $0.5 \%$ chloramphenicol topical eye drops for 3 days was the mainstay treatment for the patient. The alleviation of symptoms was however not found. Dexamethasone and neomycin eye drops shows no sort of improvement. Therefore, corneal ulcer got detected when he further decided to go to a secondary hospital for treatment. Kept as an outpatient for 2 weeks treated with oral acyclovir and $0.5 \%$ moxifloxacin eye drops. The unimproved lesion leads to further admit and treatment of the respective patient with fortified cefazolin and gentamicin eye drops. At this hospital he was diagnosed with HIV infection for the very first time. Though, the clinical condition was quite unsatisfactory. Further he got referred to the Ching Mai University Hospital's ophthalmological department. Symptoms like photophobia, ocular pain, decreased vision was reported as a complained by the patient. Best corrected visual acuity (BCVA) was 6/36 in the right eye and no light perception in the left eye. Under slit lamp examination Brownish pigmented lesion on epithelial surface and in the anterior stroma of the temporal site of the right cornea were found in his right eye along with A whitish infilteration with a feathery edge. Decreased corneal sensation along with anterior chamber reaction, hypopyon, corneal thinning, severe conjunctival injection and epithelial defect is being observed. The corneal lesion was scraped for gram staining, pottasium hydroxide examination, culture for bacteria and fungus and calcoflour white staining was performed after the patient is being treated as an inpatient. Identification of gram staining was not remarkable. Numerous large septate hyphae get shown after KOH examination and calcofluor white staining. HIV antibody test was positive,CD4 was $131.9 \%$ and fasting blood sugar was normal.

5\%netamycin eye drops were prescribed to his right eye hourly, whereas fortified cefazolin, fortified gentamicin eye drops and acyclovir were discontinued, $1 \%$ atropine kiand $0.5 \%$ levofloxacin eye drops were administered to his right eye 4 times a day with oral itraconazole (100 mg) 2 tablets a day, and vitamin C ( $500 \mathrm{mg}$ ) 1 tablet twice a day. The functioning of liver was closely monitored during the process.

Investigation and management of HIV infection was advised supported by an infectious disease specialist. Investigation of opportunistic infection occured. Cryptococcal titer antigen CSF was negative but Cryptococcal antigen serum is positive. Treponema pallidum hemagglutination test got negative, The chest X- Ray found remarkable, The Hepatitis B surface antigen found negative and the venereal disease research laboratory was nonreactive. The itraconazole (100 mg)got prescribed by the infectious disease specialist 2 tablets twice daily for Cryptococcal infection prophylaxis and 2 tablets daily of sulfamethaxazole / trimethoprim (400/80 $\mathrm{mg}$ ) for Pneumocystis caribou pnemonia prophylaxis.

The lesion seemed to be unresponsive to $0.5 \%$ netamycin and after 1 week of treatment the patients clinical condition did not improve. The overlying epithelial defect and corneal plaque got increase in size along with edge of the infilterate remained dense.. 5 weeks seem to have no change in medication. The numerous unidentified dematiaceous fungi got revealed by the consequence of previous fungal culture. Therefore, after 2 weeks of admission the corneal scraping was repeated for PCR analysis and identified as $E$. rostratum. The patient got discharged with $2 \%$ ketoconazole eye drops to the right eye 4 times a day, $1 \%$ atropine eye drops to the right eye 2 times per day, and $0.5 \%$ levofloxacin eye drops to the right eye 4 times per day after 2 months of admission.

Antifungal therapy was switched from $5 \%$ netamycin to $2 \%$ ketoconazole eye drops to the right eye every 2 hours for 2 weeks due to the patients financial problems, with $1 \%$ atropine eye drops to the right eye 2 times daily. Other medications remained unchanged including subconjunctival injection of fluconazole $(0.5 \mathrm{mg})$ every other day for 2 weeks. No AC inflammation and recurrence infilteration occur, the lesion got improved and gets replaced by a corneal scar after 4 weeks of discharge.

No light perception occurs in the left eye and BCVA gets improved to $6 / 12$ in the right eye.

\section{Safety considerations}

The safety considerations of the concern subject has been taken into formeost priority. All the medical procedures along with the medications provided are quite appropriate and are prescribed under the supervision of ophthalmologist and health care profes- 
sionals which were found to be very much appropriate and keeping the safety considerations of the concern subject under foremost priority.

\section{Follow - up}

The congenital blindness in his left eye leads to Pthisis bulbi. He was treated with eye irrigation and $0.5 \%$ chloramphenicol topical eye drops for 3 days at the primary hospital. Infilterationed by a foreign body thought to be an insect while riding a motorcycle 1 month prior to be seen.

He went to a primary hospital and was treated with eye Irrigation and $0.5 \%$ chloramphenicol topical eye drops for 3 days.

He got diagnosed with the corneal ulcer at the secondary hospital.

There he was treated with viral keratitis with oral acyclovir and $0.5 \%$ moxifloxacin eye drops for keeping the patient follow up for 2 weeks as an outpatient.

The treatment got continued with cefazolin and gentamicin eye drops when the lesion did not get improved and he get diagnosed with HIV for the very first time at this hospital.

He further got referred to ophthalmological department of Ching Mai university hospital when the condition gets worse.

After looking at all the parameters of signs and symptoms shown by the patient he was prescribed an appropriate medication for 1 week

After keeping the patient follow up for 1 week the patient clinical conditions did not improve and the lesion seen to be unresponsive to $0.5 \%$ netamycin.

Therapeutic debridement and subconjunctival injection of fluconalzole were performed every other day.

PCR analysis got repeated for corneal scraping and after two weeks got diagnosed as E. rostratum.

The corneal scar got replaced without an overlying superficial corneal plaque or epithelial defect and lesion did not get improved after 5 weeks of treatment.

At every 2 hours the anti fungal therapy get switched from 5\% netamycin to $2 \%$ ketoconazole eye drops for 2 weeks due to pa- tients financial problems. For every other day for 2 weeks medications including subconjunctival injection of fluconazole $(0.5 \mathrm{mg})$ remain unchanged. At $2 \%$ ketoconazole eye drops to the right eye 4 times per day, $1 \%$ atropine eye drops to the right eye 2 times per day and $0.5 \%$ levofloxacin eye drops to the right eye 4 times per day he got discharged. The lesion got improved after 4 weeks of discharge with no AC inflammation and recurrence infiltration, however the corneal scar replaced the lesion. The left eye perceives no light perception and BCVA improved to 6/12 in the right eye.

\section{Data management and statistical analysis}

The creative commons Attribution Non - commercial 4.0 International license ( CC BY -NC) gets the licensed for this article. S. Karger AG Basel Published the article as The authors (c) 2019 www.karger.com/cop DOI : 10.1159/000499688

\section{Quality assurance}

The Quality of the article is being assured as the content of the study is accurate and on the basis of a case report.

The safety of the article is being kept at foremost priority as it is published by Karger open access publishing authorization.

\section{Expected outcomes of the study}

The main outcomes of the study is to evaluate the prognosis of a patient who is suffering from fungal corneal ulcer by Exserohilum Rostratum who is also HIV patient.

The study also tells about the pathogenic nature of Exserohilum species.

It tells about the appropriate medication which is suppose to prescribe at the time of an ocular infection with Exserohilum species.

Dissemination of results and publication policy

Karger open access is the publication policy which publishes the study.

(C) 2019 the author(s) Published by S. Karger AG, Basel www. karger.com/cop The creative commons attribution Non - commercial 4.0 gets the licensed for this article ( CC BY -NC) Thantawat Theeranan M.sc gets the acknowledgement by the author for the English manuscript.

\section{Duration of the project}

An ideal Duration of the project is consider as slightly above 2 months time considering all the follow up visits by the patient. 
Beside this 2 months time nearly 1 month time is being considered to check the prognosis, whether the patient is being fully recovered by the fungal infection and to check the effective rate of the prescribed drugs.

\section{Collaboration with other scientists or research institutes}

Some of the investigators and scientists involve in this study are as follows :

- Winai Chaidaroon.

- Mitt Phaocharoen.

- Titipol Srisimboon.

- Nongnuch Vanittanakom.

- Ching Mai university's Department of ophthalmology, Faculty of medicine makes contribution in the research work.

- Ching Mai university's Department of Microbiology, Faculty of medicine, is involved in the research work.

- These are some of the research institutes involve in This study.

Funding sources

No financial support is receive from the public by the author for this research work.

\section{Statement of ethics}

The declaration of Helsinki along with the tenets gets compiled up for the study. The research ethics committee of the institute approved the study protocol on human research. For the publication of this case report the written informed consent was obtained from the respective patient and any accompanying images.

\section{Author Contributors}

Nutt Phaocharoen and Winai chaidaroon makes contribution regarding study method and design.

- Acquisition, specimen analysis and interpretation of data is done by all the authors.

- Winai Chaidaroon and Nutt Phaocharoen plays an important role in drafting of the manuscript.

- Winai Chaidaroan and Nutt Phaocharoen did the critical revision of the manuscript for the intellectual content.

- Winai Chaidaroon did the study supervision [1-5].

\section{Pictures representing the actual case study}

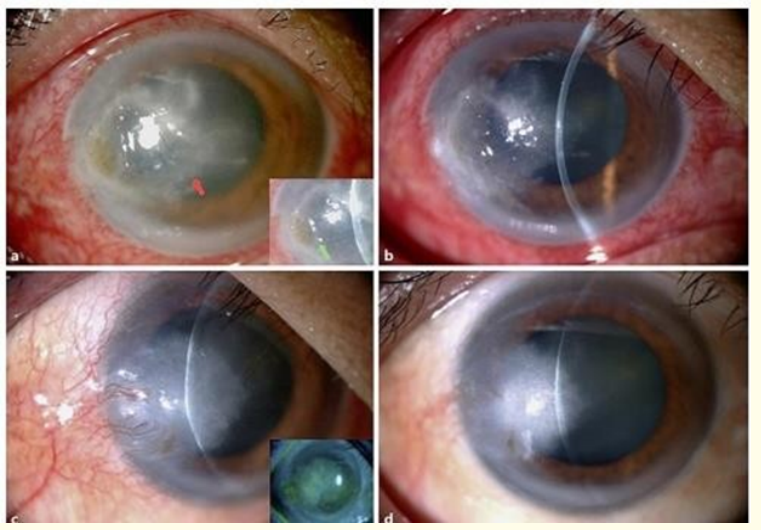

Figure 1: Photographs of the right eye before and after treatment a. At the first visit,(a ) A brownish pigmented lesion on the epithelial surface and anterior stroma also a whitish infilteration with a feathery edge were presented at cornea at the first visit.(b) Corneal plaques and dense infilterate were found one week after medical treatment.(c) The lesion was replaced by a corneal scar without an overlying superficial corneal plaque and epithelial defect after five weeks of medical treatment. (d) After 2 months of treatment the lesion became a corneal scar.

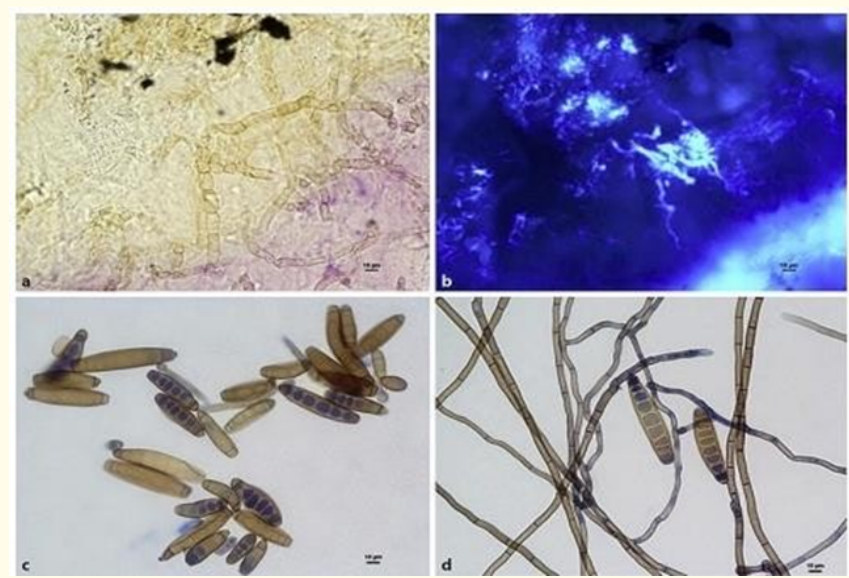

Figure 2: $\mathrm{KOH}$ examination (a) and calcoflour white staining (b) showed numerous large septae hyphae (c),(d) Fungal culture revealed numerous dematiaceous fungi of Exserohilum Rostratum (asexual state). 


\section{Bibliography}

1. Sutton DA., et al. The Disseminated phaeohypomycosis is the review of an emerging mycosis (2002).

2. Yaniv I., et al. Avrahami G made the reference for the conclusion of exserohilum as an emerging human pathogen.

3. Prajna VN., et al. Made the reference for the conclusion of clinical signs in dematiaceous and hyaline fungal keratitis.

4. Rinaldi MG., et al. Made the reference for the conclusion of emerging agents of phaeohypomycosis as pathogenic species of bipolaris and exserohilum.

5. Sutton DA and Revanker SG. Made the reference for the conclusion of Melanised fungi in human disease.

\section{Assets from publication with us}

- Prompt Acknowledgement after receiving the article

- Thorough Double blinded peer review

- Rapid Publication

- Issue of Publication Certificate

- High visibility of your Published work

Website: www.actascientific.com/

Submit Article: www.actascientific.com/submission.php

Email us: editor@actascientific.com

Contact us: +919182824667 